\title{
MWSTAT: A MODULATED WEB-BASED STATISTICAL SYSTEM
}

\author{
Francisco Louzada ${ }^{1 *}$ and Anderson $\mathrm{Ara}^{2}$
}

Received February 17, 2017 / Accepted April 20, 2018

\begin{abstract}
In this paper we present the development of a modulated web based statistical system, hereafter MWStat, which shifts the statistical paradigm of analyzing data into a real time structure. The MWStat system is useful for both online storage data and questionnaires analysis, as well as to provide real time disposal of results from analysis related to several statistical methodologies in a customizable fashion. Overall, it can be seem as a useful technical solution that can be applied to a large range of statistical applications, which needs of a scheme of devolution of real time results, accessible to anyone with internet access. We display here the step-by-step instructions for implementing the system. The structure is accessible, built with an easily interpretable language and it can be strategically applied to online statistical applications. We rely on the relationship of several free languages, namely, PHP, R, MySQL database and an Apache HTTP server, and on the use of software tools such as phpMyAdmin. We expose three didactical examples of the MWStat system on institutional evaluation, statistical quality control and multivariate analysis. The methodology is also illustrated in a real example on institutional evaluation. A MWStat module was specifically built for providing a real time poll for teacher evaluation at the Federal University of São Carlos (Brazil).
\end{abstract}

Keywords: Online Survey, Statistical System, Real Time Results, Statistical Methods.

\section{INTRODUCTION}

For decades the pattern of rationality based on statistical analysis has experienced slowness, which begins with the data collection, passes by the statistical analysis itself, and ends up in the final presentation of the results obtained in form of a report. For instance, in traditional data collect research there is a gap in time between the application of a questionnaire, the obtainment of the answers of respondents, the application of statistical techniques and the visualization of informative reports on the final results, i.e., possibly a slow process that often may involve a high cost. On the other hand, on account of internet democratization, online surveys are becoming

\footnotetext{
*Corresponding author.

${ }^{1}$ Instituto de Ciências Matemáticas e de Computação, Universidade de São Paulo - USP, São Carlos, Brazil. E-mail: louzada@icmc.usp.br

${ }^{2}$ Departamento de Estatística, Universidade Federal da Bahia - UFBA, Salvador, Brazil. E-mail: anderson.ara@ufba.br
} 
widely used. In an informal survey over the network, Kaye \& Johnson (1999) identified over 2000 surveys in 59 different areas that benefit from the technology focused on online questionnaires. Solomon (2001), Fricker et al. (2005) and Cox \& Cox (2008), amongst others, show internet based surveys offer significant advantages over traditional survey techniques. However, despite the wide use of online research, the gap in time between the begin of a survey and the final presentation of the statistical analysis results remains. Thus, there is a real need for a environment which facilitates the application of a survey while directly connecting the responses in a customizable real time informative report in order to quickly provide all the information generated by the survey.

Over the world wide web we can find some environments that organize national statistics datasets, such as the Virtual Statistical environment housed on:

http://www.virtualstatisticalenvironment.org/,

as well as, there are environments which collect online data focusing on creating and publishing online surveys, such as the SurveyMonkey (http: / /www . surveymonkey.com/) and QuestionPro (http: //www. questionpro.com/). It is also easily found statistical systems for specific statistical analysis, such as Young Tung \& Schuenemeyer (1991), Krtolica et al. (1991), Hatanaka \& Yamada (2003), Analytica (http://www. lumina.com/), and Plug\&Score (http://plug-n-score.com), just to name a few.

Moreover, some online statistical environments are capable to produce some simple plots, frequency tables, cross-tabulation and summary measures. SOCR (Dinov, 2006) (http: / /www . socr . ucla . edu /) proposes a suite of Java applets for statistical online computation, visualization, analysis and virtual experimentation, EasyCalculation environment (http://easycalculation.com/) is a free online math website which helps users to learn mathematics and statistics. The above environments cannot carry out customized statistical analysis which may involve more sophisticated procedures, such as a regression analysis, a multivariate analysis, quality control charts, among others.

Other environments such as the R-PHP (Mineo \& Pontillo, 2006) (http://dssm.unipa.it/ R-php/), RStudio (RStudio, 2012) and rApache (Horner, 2012) allow the utilization of R software directly online, that may be installed on any server. These environments have a sophisticated communication between the $\mathrm{R}$ software and servers, but do not interact dynamically with users who do not have knowledge of the $R$ language. The environment JStatCom (Krätzig, 2007) defines some classes to connect existing math libraries (Ox, Matlab or R programming languages) with Java client. The Rweb environment (Banfield, 1999) has three different versions, the first allows to type the code, click the submit button and a page with the results is returned, the second is based on Javascript procedures and the third is designed as a point click interface that can be used in introductory statistic courses. Also, Online Analytical Statistical Information System (OASIS, 2015) is a online system that provides some sophisticated and public analysis of health and social science data. And, R-fiddle (R-FIDDLE, 2015) provides a free environment to write, run and share R-code right inside the browser.

In Operations Research, the use of softwares is quite indispensable. Even though there are several traditional software as LINDO, AIMMS, GAMS, amongst others. Various authors have proposed their own softwares based on some general programming languages. For instance, APSIM (McCown et al., 1996) is an offline software developed in C++ and Visual BASIC to models of crop and pasture production and performs dynamic simulations. SIAD (Meza et al., 2005) is an offline software encoding in Delphi's with 
the Simplex algorithm to solve Linear Programming Problems. MEIGO (Egea et al., 2014) is an offline $\mathrm{R}$ and Matlab optimization toolbox that implements metaheuristics. Some other examples can be found in McCown et al. (1996), Kolisch \& Sprecher (1997), Gondzio et al. (1995), Fernandes et al. (2007), Schramm $\&$ Morais (2012) and Gould et al. (2015). In this sense, to the best of our knowledge, we do not know any online software capable to perform data analysis with real time exposure of the results.

In the present paper we built the MWStat system environment which is based on a scheme of devolution of real time results, accessible to anyone with internet access. In other words, these procedures are focused on how to build a user-friendly interface and how to relate any website with the R software to generate dynamic results for any online purposes. The procedures exposed in this paper may be easily applied by statisticians with a basic knowledge of web programming. The structure is accessible and built with an easy interpretation language (PHP) and strategically applied in online applications. This procedure can be considered an system, since it replaces the manual collecting data and is able to expose more targeted results to real problems.

The MWStat relies on the relationship of several free open-source languages, namely, PHP (http//: www.php.net), R (http//: cran.r-project.org), MySQL database (http//: www.mysql. com) and an Apache HTTP server (http//: www . apache.org), the latter for hosting and interpreting other languages. Moreover, we used the phpMyAdmin interface (http/ / : www . phpmyadmin.net) as an auxiliary tool, related to the administration of MySQL database using PHP language. These technologies were gathered in a LAMP server (Linux, Apache, MySQL and PHP), which is a popular solution of free open source software to build a viable web server of general purpose with a low costing structure and high performance (Neiderauer, 2004).

Combining all the technologies above, the MWStat can perform online collection and data analysis, and the obtained results are provided in real time, depending only on the processing time of the statistical analysis to be considered. Moreover, the MWStat is also very flexible, since it is completely customizable and it can be built into independent modules, according to the user needs.

The main objective of the present paper is to present the MWStat and the softwares involved in its construction, displaying some technical procedures on how to building it. Following this paper, anyone with some computational knowledge may build a web based statistical system environment that performs online statistical analysis for any purposes. The versions of the software used in this work are provided, but the same procedures can be extended to different versions or even other operating environment platform and database software. We provide the basic codes for the implementation of the R environment on any LAMP server, but more detailed codes are available in our homepage (http: / /www.mwstat.com) as well as in the Supplementary Material.

In Section 2 exposes the softwares used for building the MWStat and their considered versions as well as some comments around the instalation procedures. In Section 3 we present examples of the MWStat implementation for event evaluation, as well as for two more areas, statistical quality control and multivariate analysis, those can be accessed in the MWStat homepage through login and password provided. Moreover, a fourth example is also provided on institutional evaluation, which has been used in several applied researchers in Brazil. We finish the paper with some final comments in Section 4, where we also present the web based statistical system homepage and the various applied research developments based on its environment. 


\section{SOFTWARES APPLIED FOR BUILDING THE MWSTAT ENVIRONMENT}

In this section, we present a list of the auxiliar softwares used in the construction of the MWStat. Essentially, they are free softwares which can be easily found on the web.

- Linux Ubuntu Server 9.10 (or higher): Ubuntu is a complete Linux operating environment, completely free, with great practicality, configuration and use (Tarng \& Liou, 2006). The installation file of this operating environment is available on website http: / / www . ubuntu.com, where the image of its installation $\mathrm{CD}$ can be downloaded.

- R software: computing environment for the performance of statistical analysis and graph building. It compiles and runs on a wide variety of UNIX platforms, Windows and MacOS. In our case, we used the $\mathrm{R}$ implemented in UNIX through a Linux server.

- MYSQL 5.0 (or higher): database management environment (DBMS) that uses the SQL (Structured Query Language) as interface. It is currently one of the most popular databases, with over 100 million installations worldwide.

- PHP 5.0 (or higher): (James \& Ware, 2003) acronym for Hypertext Preprocessor, is a language for creating script directly into the server designed specifically for the web. Within an HTML page, PHP codes can be executed every time the page is visited. This code is interpreted on the web server and generates HTML viewing or other display type. Below are listed some advantages of PHP: high performance; interfaces for many different database environments; integrated libraries for many common tasks from the web; Low cost, Easy to learn and use, portability, availability to source code. Version 5.0 was developed to improve to Object Oriented Programming and is available now in version 5.2.13 (http: / / www . php. net).

- phpMyAdmin 2.7.0-pl2: (or higher): computer program developed in PHP to administer MySQL over the Internet. From this environment you can create and remove databases; create, delete and modify tables; insert, delete and edit fields, execute SQL code and manipulate key fields. For this paper, we used implemented features and bug fixes up to version 2.6.2 (http: //www.phpmyadmin.net).

These softwares should be installed in order to build a LAMP server capable to gathering, storage data and show the results through the Apache, MySQL and PHP as well as perform statistical analysis and process the data through the R Software. Figure 1 shows the overall MWStat environment server structure.

The Appendix describes the implementation procedures focused on listing the procedures for creating the MWStat environment, as well as the software installation, settings and programming, at the expense of displaying the best hardware configuration for a computer (server) to implement the system, which is out of the scope of the paper.

\section{Some Applications}

In this section, we display three general applications of our system. The first, second and third examples of applications of the system for event evaluation, quality control and multivariate analysis, respectively. They can be accessed online by the readers. Moreover, the first and third o applications can be totally reproduced in a standalone fashion by downloading the Supplemental Material and codes from http: //www. mwstat. com. The fourth application is an institutional evaluation which was carried out on an undergraduate curse in Statistics from the Universidade Federal de São Carlos, Brazil. 


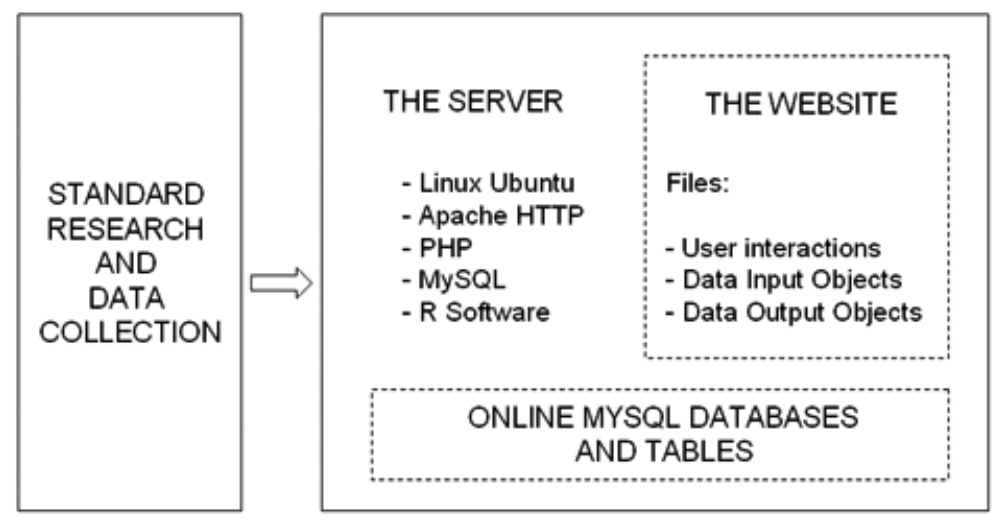

Figure 1 - General structure of the system environment.

\subsection{Some introductory examples}

In this section, we display more three general didactical applications of the MWStat. The first one is based on a dynamic event evaluation which allows to measure the quality of scientific events, such as, conferences, symposia, meetings, workshops, among other. This example consists in 11 questions about academic purposes in a generic event. Participants answer an online questionnaire and an instant online report is provided in real time. The files and their relationship for this example are shown in Figure 9. The files may be downloaded from http: / /www. mwstat. com in a standalone fashion or can be found in the Supplementary Material, in the sense that an interested reader can easily reproduce the overall example. The Figure 2 shows the online questionnaire and Figures 3 and 4 show a part of the instant report.

The second application is focused in statistical process control, the default dataset was taken from Montgomery's book (Montgomery, 1991). It consists of 40 samples of size 4 to control of the diameter of the piston rings. The Figure 5 shows the online reports with a default dataset in SPC analysis.

The third example is based on a principal component analysis, the default dataset (Drapper \& Smith, 1966) is composed by a sample of 4 variables about 21 days of operation of a plant oxidizing. The Figure 6 shows the online report with a default dataset for PCA analysis. The files may be downloaded from http: / / www . mwstat. com in a standalone fashion or can be found in the Supplementary Material, in the sense that an interested reader can easily reproduce the overall example.

All examples present above can be accessed online from the address http://www. mwstat. com in the article examples link. To access the examples enter the website and provide the password HJT534; the uppercase letters must be maintained. In the event evaluation example, the readers may answer the online questionnaire and access the entire instant report. The password to access this questionnaire is mwstat. According to the present exemplification, only one user was established, but an indefinite number of users could be set up. In the other examples the readers may enter their own dataset or use a given default dataset available in the environment. 


\section{EVALUATION SYSTEM}

\section{PROFILE}

Training area: $\langle-$ Select- $>$

Your highest academic degree:
Finishing Graduation
- Finishing Master degree
- Finishing Doctorate
- Finishing Post Doctorate
Other. Which one?

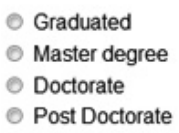

Gender: <-Select ->

Age: years

Where from: $\langle$-Select $\rangle$ -

\section{Is your highest academic degree in Statistics?}

Yes $\odot$ No

\section{SPECIFIC QUESTIONS}

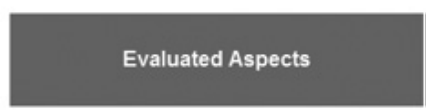

1) Technical level of theme sessions*

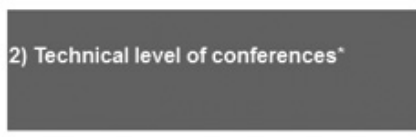

3) Technical level of short courses*

\section{4) Lecturer Commitment}

5) Infrastructure available

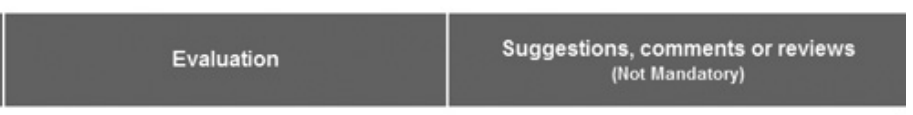

\section{$\bigcirc 1 \bigcirc 2 \bigcirc 3 \bigcirc 4 \bigcirc 5 \bigcirc 6 \bigcirc 7 \bigcirc 8 \bigcirc 9$}

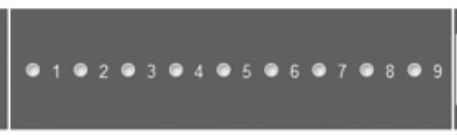

010203040506070809
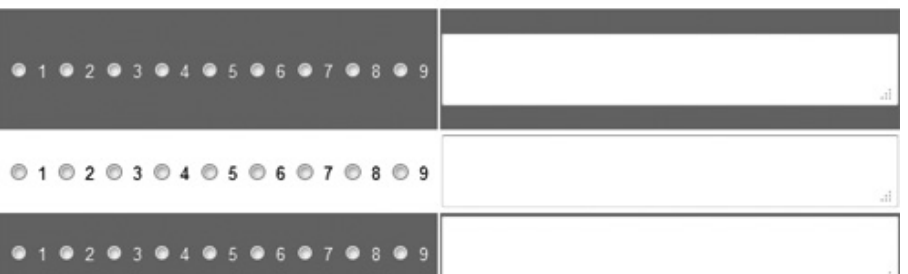

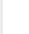

$102 \bigcirc 3 \bigcirc 4 \bigcirc 5 \bigcirc 6 \bigcirc 7 \bigcirc 809$

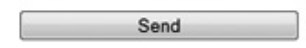

Figure 2 - Online questionnaire for the event evaluation example.

\subsection{Institutional Evaluation Example}

The fourth example is related to an institutional evaluation. The main purpose of institutional evaluation is to support the university's commitments to academic and operational excellence through the collection, analyses and reporting of diversified data. The MWStat environment was used with the purpose of evaluating lectures and general aspects of a particular department within a Brazilian university. The environment was tested on a stratified sample of 165 students of an undergraduate statistics course. Thus, each randomly chosen student received an email containing an explanatory text followed by the website address and a 


\section{Personal Traits}

Overall:

- Number of Participants: 3

- Age (mean): $\mathbf{3 4}$

- Academic degree in Statistics:

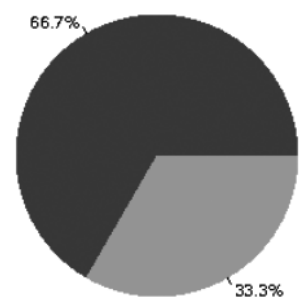

Legend: - Statistics
Others
Participants per continent:

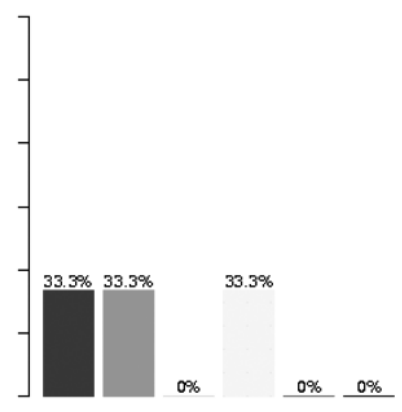

Legend:

- North America - South America Europe
Africa
- Asia

- Asia

Figure 3 - A part of the Instant personal traits report for the event evaluation example.

random password, with which it was possible to access the restricted area designated to the students. The password was randomly generated and encrypted in the database.

After each student answered the questionnaires designated to them, the environment sent a new e-mail thanking and confirming the successful storage of the responses. The answers are properly inserted into a MySQL database that safely only the database administrator (researcher) has access.

After each student performed his responses successfully, the homepage of the website is updated and a link to the evaluation results is provided. An instantaneous report can be viewed throughout all the evaluation.

In the authentication area, programmed in PHP, the login and password of the individual are verified and, furthermore, the level of hierarchy of information compatible with his status is identified. In other words, how much information is provided to him. For instance, students may have a different level of information in comparison with the lectures they evaluated. In terms of illustration, let us consider that the login was made by the director for undergraduate studies in statistics. The director has access to the overall online report of his departmental lectures evaluation. An illustration of such report is shown in Figure 7. The report displays a principal component analysis, and the general evaluation of each particular aspect. However, other statistical methodologies can be straightforwardly considered.

For sake of comparison we tried, without success, to consider the most known online statistical environments with the purpose of evaluating lectures and general aspects of the particular problem treated in this section. Survey Monkey and QuestionPro have some free licenses able to perform one survey that contains at most ten questions and one hundred responses, more advanced surveys can be carried out through 


\section{Overall Evaluation}

Generated in 06-22-2015

Traffic-line satisfaction :

\begin{tabular}{lllllllll}
1.0 & & 2.6 & & 4.2 & & & & \\
\hline & Awful & & Bad & & Reqular & Good & Excellent \\
\hline
\end{tabular}

Aspects

Accumulated satisfaction

Thematic sessions

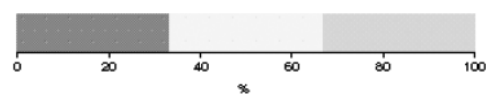

Conferences

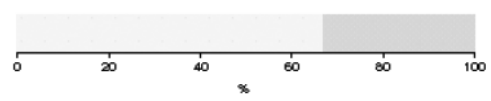

Short courses

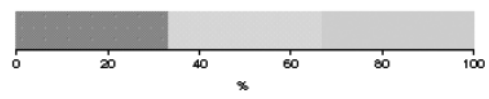

Speakers

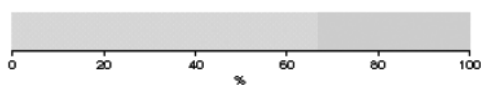

Figure 4 - Another part of the Instant personal traits report for the event evaluation example.

payment; remembering 165 students were sampled. Besides, their statistical analysis are based only on descriptive statistics and basic graphs; a principal component analysis could not be considered. SOCR has many possible statistical analyses but the environment is restricted by the developer's Java applets and it is not customizable. R-PHP does not have any online procedure enabling data collection and analysis in real time.

Thus, each in its turn is overcome by the MWStat. Through the steps exposed in this paper, the MWStat arises as a environment which is able to carry out any number of online surveys, with unlimited number of questions and responses. Besides the MWStat performs any customized statistical analysis that may involve more general sophisticated procedures. With our open source technology it is possible to perform any kind of data collection and analysis in virtual environments, allowing the monitoring of the real-time results.

\section{FINAL COMMENTS}

This paper discuss a current technological trend based on statistical calculations directly online, and displays the necessary tools to build a module of the MWStat, a dynamic environment applied to online research. The MWStat results from the relationship of three programming languages (PHP, MySQL and R), and it may be considered for performing many statistical tasks within a web page structure. Our focus here is to display the necessary procedures so that the MWStat may be implemented and disseminated in other servers. The development of other aspects such as web forms is linked to the experience of the programmer who will use the server displayed here. 


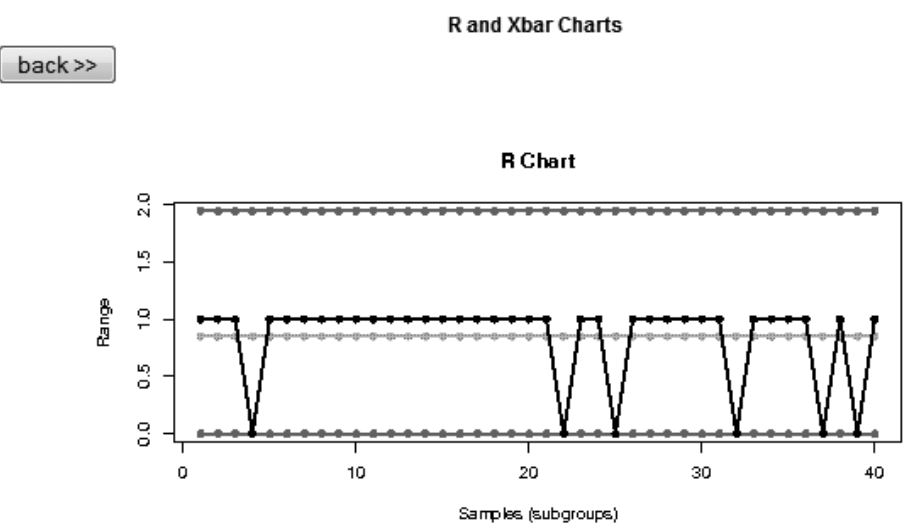

Xbar Chart

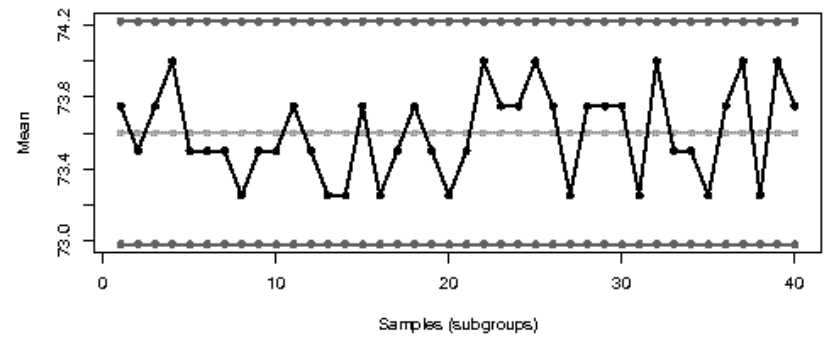

Figure 5 - A online report of the statistical control process example.

There is plenty of room for future developments of the MWStat. We believe almost all statistical techniques can be implemented by using the strategy proposed in the paper shifting the statistical paradigm from offline to online analysis, accessible to anyone with only internet access. For instance, we envisage new MWStat modules on methodologies such as time series for financial online analysis, online statistical quality control, parametric and non-parametric bioequivalence tests, and so on. In general terms, by means of any method implemented on the R software, the MWStat can perform online collection, data analyses, results and even reports, providing real time up-to-date statistical methods available online to practitioners and researchers.

A possible challenge is to consider the MWStat for educational purposes, since it may provide a straightforward path for learning statistics, without the need of installing any software but only an internet access.

Figure 8 presents the MWStat homepage ( http://www. mwstat.com), where the presented examples may be tried as well as the entire codes of the first and second examples may be downloaded as a supplementary material, providing an straightforward way of interested readers to reproduce the statistical analysis presented here in their own servers, as well as develop their own $\mathrm{R}$ web based statistical applications.

The MWStat has been used in several applied researchers in Brazil and abroad. We cite the development of the module SAO-Docentes (in Portuguese, SAO is the acronym for "Sistema de Avaliação Online" and Docentes denotes lectures), which was specifically built for providing a real time poll for teacher evaluation 
In this example we only consider the PCA method by formulation through correlation matrix .

The two firsts PCA explain $93 \%$ of data variation.

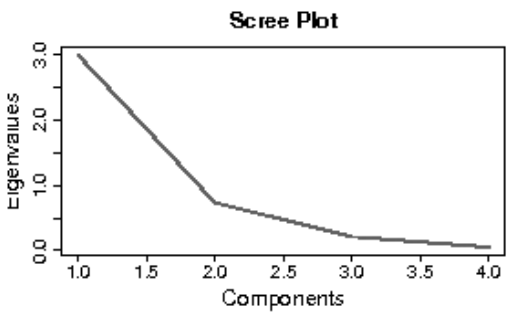

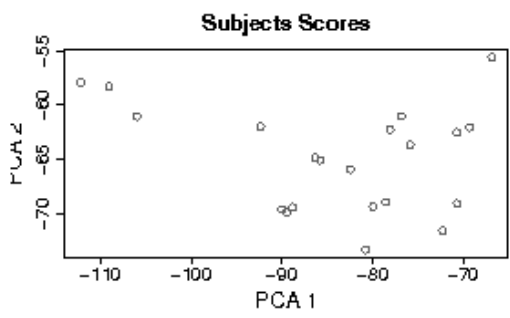

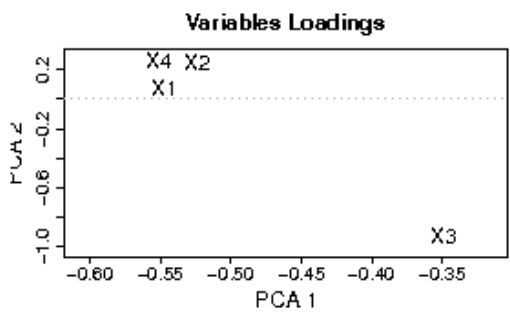

Figure 6 - A online report of the principal component analysis example.

at the Federal University of São Carlos (Brazil). During the years of 2010-2016, the SAO-Docente was answered for more than 20,000 students for teacher evaluation of more than 2,000 university courses. The module SAO-Egressos (in Portuguese, Egressos denotes graduates or egress students), which was built to provide information on an overall evaluation of the Federal University of São Carlos (Brazil) by its egress students. Over 2011-2014, more than 4,000 ex-students were exposed to the SAO-Egressos. Some reports (in Portuguese) may be found in the site www. cpa. ufscar.br. Further, the MWStat has been used for business satisfaction surveys. For instance, we cite a hotel poll conducted for a Brazilian hotel group in 2009 and 2010, a business poll conducted for a Brazilian accounting company in 2009 and 2011, a question system for a Business and Commercial Chamber of two counties in 2014. The MWStat has also been used as part of the methodological structure of statistical analysis in masters and doctorate thesis, such as the "The national policy on technical assistance and rural extension: Perceptions and Trends" by Pettan (2010) and "Perception of the architectural work space for the university community: the UFSCar case study" by Salvador (2011). Moreover, the MWStat has been successfully applied for more than 50 congresses evaluations in Brazil, Portugal and Peru. We point out some of them such as the 54th Annual Meeting of the Brazilian Region of the International Biometrical Society and the 13th Symposium on Applied Statistics to Agronomic Experimentation, both held in São Carlos, Brazil, in 2009, the 3td School of Sampling and Research Methodology and the 2nd International Workshop on Surveys for Policy Evaluation, both held in Juiz de Fora, Brazil, in 2011, the 20th, 21st and 22nd National Symposium of Probability and Statistics, held respectively in João Pessoa, Natal and Porto Alegre, Brazil, in 2012 and 2014, the 60th ISI World Statistics Congress (WSC), held in Rio de Janeiro, Brazil, July 2015, the 35th and 36th Congresso Nacional de Matemática Aplicada e Computação, held in Natal and Gramado, Brazil, in 2014 and 2016. 


\section{REAL TIME REPORT}

Note: Below please find the real time analysis generated by the web stat. These are some statistics which show the progress of the lecturer's evaluation until now.

Report's structure:

This Principal Component Analysis considers only two scores (XPC1 and XPC2) according to the lecturer's evaluation in the conception of their students.

\begin{tabular}{|l|c|c|}
\multicolumn{3}{c}{ Some Aspects Evaluated: } \\
\hline Aspects & Mean: & Evaluation \\
\hline Question Stimulation & 7.7 & Excellent \\
\hline Receptivity & 7.6 & Excellent \\
\hline Knowledge Encouragement & 4.4 & Regular \\
\hline Respect & 6.8 & Good \\
\hline Bibiliography & 7.9 & Excellent \\
\hline Incentives to research & 4.7 & Regular \\
\hline Knowledge & 5.4 & Regular \\
\hline Clarity & 3.6 & Bad \\
\hline Association with real problems & 7.0 & Good \\
\hline Interdisciplinarity & 8.6 & Excellent \\
\hline Motivation & 7.2 & Good \\
\hline Preparation of Lessons & 6.6 & Good \\
\hline
\end{tabular}

Qualitative assessments are built to better understand the results, which are classified in the traffic-line assessment presented below:

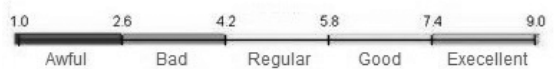

Principal Component Analysis:

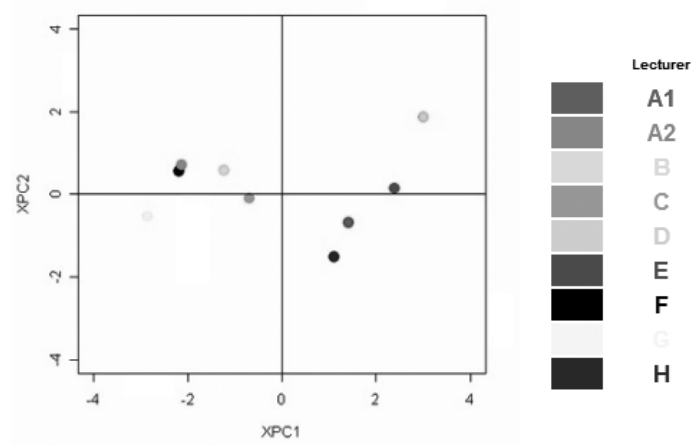

XPC1 (abscissa): score of respect, interest, competence, ethic, objectivity and clarity of the explanations. This can be interpreted as a score of friendliness of the lecturer. The smaller the component, the friendlier the professor is to his students.

XPC2 (ordinate): score of scientific encouragement and methodological organization of the lecturer. This can be interpreted as a score of scientific knowledge.

Figure 7 - Online report on view at course coordinator.

\section{ACKNOWLEDGMENTS}

The researchers of Francisco Louzada and Anderson Ara are supported by the Brazilian organisations CNPq and FAPESP.

\section{REFERENCES}

[1] BAnfield J. 1999. Rweb:Web-based Statistical Analysis. Journal of Statistical Software, 4: 1-15.

[2] Cox JB \& Cox KB. 2008. Your Opinion, Please: How to Build the Best Questionnaires in the Field of Education. Corwin Press, New York. 2nd Edition. 136 pp.

[3] DINOV ID. 2006. Socr: Statistics online computational resource. Journal of Statistical Software, 16: $1-20$.

[4] Drapper NR \& Smith H. 1966. Applied Regression Analysis. John Wiley \& Sons, New York. 1st Edition. 407 pp.

[5] Egea JA, Henriques D, Cokelaer T, Villaverde AF, Macnamara A, Danciu D.-P, BANGA JR \& SAEZ-Rodriguez J. 2014. Meigo: an open-source software suite based on metaheuristics for global optimization in systems biology and bioinformatics. BMC bioinformatics, 15(1): $136 \mathrm{pp}$. 


\section{mwstat.com}

Current modules
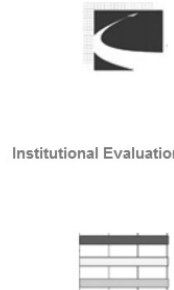

Event Evaluation System

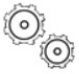

Control Quality System

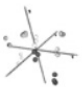

Principal Component Analsys
Send us your work and become part of our portal. Your module will be joined to other modules that use the web-stat technology around the world.

Send an email to: anderson@ufscar.br with the following information:

-URL

- Authors

- Title of Work

- Statistical technique involved

- Logo of your website

Figure 8 - Website of the MWStat where possible developers may provide their statistical analysis modules for general users.

[6] Fernandes S, Captivo ME \& Climaco JA. 2007. Sabiloc: um sistema de apoio a decisão para análise de problemas de localização bicritério. Pesquisa Operacional, 27(3): 607-628.

[7] Fricker S, Galesic M, Tourangeau R \& Yan T. 2005. An experimental comparison of web and telephone surveys. Public Opinion Quarterly, 69(2): 370-392.

[8] Gondzio J \& Others. 1995. Hopdm (version 2.12)-a fast lp solver based on a primal-dual interior point method. European Journal of Operational Research, 85(1): 221-225.

[9] Gould NI, ORBAn D \& Toint PL. 2015. Cutest: a constrained and unconstrained testing environment with safe threads for mathematical optimization. Computational Optimization and Applications, 60(3): 545-557.

[10] Hatanaka M \& Yamada H. 2003. Co-trending: A Statistical System Analysis of Economic Trends. Springer-Verlag, Tokyo. 1st Edition. 115 pp.

[11] Horner J. 2012. rApache: Web application development with R and Apache. http: //www . rapache.net/

[12] James DA \& DebRoy S. 2006. RMySQL: R interface to the MySQL database. R package version 0.5-11. Edition. https://cran.r-project.org/src/contrib/Archive/ RMYSQL/RMYSQL \_0.5-11.tar.gz

[13] James L \& WARE B. 2003. Open Source Web Development with LAMP: Using Linux, Apache, MySQL, Perl, and PHP. Addison Wesley. 1st Edition. 496 pp.

[14] Kaye B \& Johnson T. 1999. Research methodology: Taming the cyber frontier. Social Science Computer Review, 17(1): 323-337.

[15] Kolisch R \& Sprecher A. 1997. Psplib-a project scheduling problem library: Or software-orsep operations research software exchange program. European Journal of Operational Research, 96(1): 205-216. 
[16] KRÄTZIG M. 2007. A software framework for data analysis. Computational Statistics \& Data Analysis, 52(2): 618-634.

[17] Krtolica R, ObRadovic I \& Bozinovic R. 1991. Sfiltex: toward an expert system for time-series filter design. Journal of Applied Statistics, 18: 121-137.

[18] McCown R, Hammer G, Hargreaves J, Holzworth D \& Freebairn D. 1996. Apsim: a novel software system for model development, model testing and simulation in agricultural systems research. Agricultural systems, 50(3): 255-271.

[19] Meza LA, Biondi Neto L, Mello JCCBS, Gomes EG \& otheres. 2005. Isyds-integrated system for decision support (siad-sistema integrado de apoio à decisão): a software package for data envelopment analysis model. Pesquisa Operacional, 25(3): 493-503.

[20] Mineo AM \& Pontillo A. 2006. Using R via PHP for teaching purposes: R-php. Journal of Statistical Software, 17: 1-12.

[21] Montgomery DC. 1991. Introduction to Statistical Quality Control, 2nd Edition. John Wiley \& Sons, New York. 2nd Edition. 702 pp.

[22] Neiderauer J. 2004. Php para quem conhece php: recursos avançados para a criação de websites dinâmicos. São Paulo: Novatec Editora Ltda. $2^{\mathrm{a}}$ Edição. 480 pp.

[23] OASIS. 2015. Oasis. http: / /www. oasisdataarchive.org/

[24] Pettan KB. 2010. A política nacional de assistência técnica e extensão rural (pnater): Percepções e tendências. Ph.D. thesis, Universidade Estadual de Campinas - UNICAMP, Brazil.

[25] R-FIDDLE. 2015. R-fiddle. http: / /www.r-fiddle.org

[26] RSTUDiO. 2012. Integrated development environment for R (Version 0.96.122) [Computer software]. Boston, MA. http: / /www.rstudio.org/

[27] SAlVAdor EV. 2011. Percepção arquitetônica do espaço de trabalho pela comunidade universitária. Estudo de caso da UFSCar - Campus São Carlos. Master's thesis, Universidade Federal de São Carlos, Brazil. https: //repositorio.ufscar.br/handle/ufscar/4325. 142 pp.

[28] Schramm F \& Morais DC. 2012. Decision support model for selecting and evaluating suppliers in the construction industry. Pesquisa Operacional, 32(3): 643-662.

[29] Solomon DJ. 2001. Conducting web-based surveys. Practical Assessment, Research \& Evaluation, 7(19): $1-4$.

[30] TARng W \& Liou H. 2006. The development of an internet virtual zoo. Advanced Technology for Learning, 3(2) (E2): 69-81.

[31] Young Tung ST \& SChuenemeyer JH. 1991. An expert system for statistical consulting. Journal of Applied Statistics, 18: 35-47.

\section{APPENDIX: IMPLEMENTATION PROCEDURES}

In this appendix we show the implementation of the environment in an Ubuntu LAMP server. The Linux operating environment was installed on a specific computer, following these steps:

1. Through the installation file from the Ubuntu 9.10 Linux, we create a CD to start the computer from it, in other words, do the boot from the $\mathrm{CD}$. If the $\mathrm{CD}$ does not run automatically, we must configure the Setup (BIOS) for boot priority.

2. Choose the preferred language (shortcut to select language: F2). 


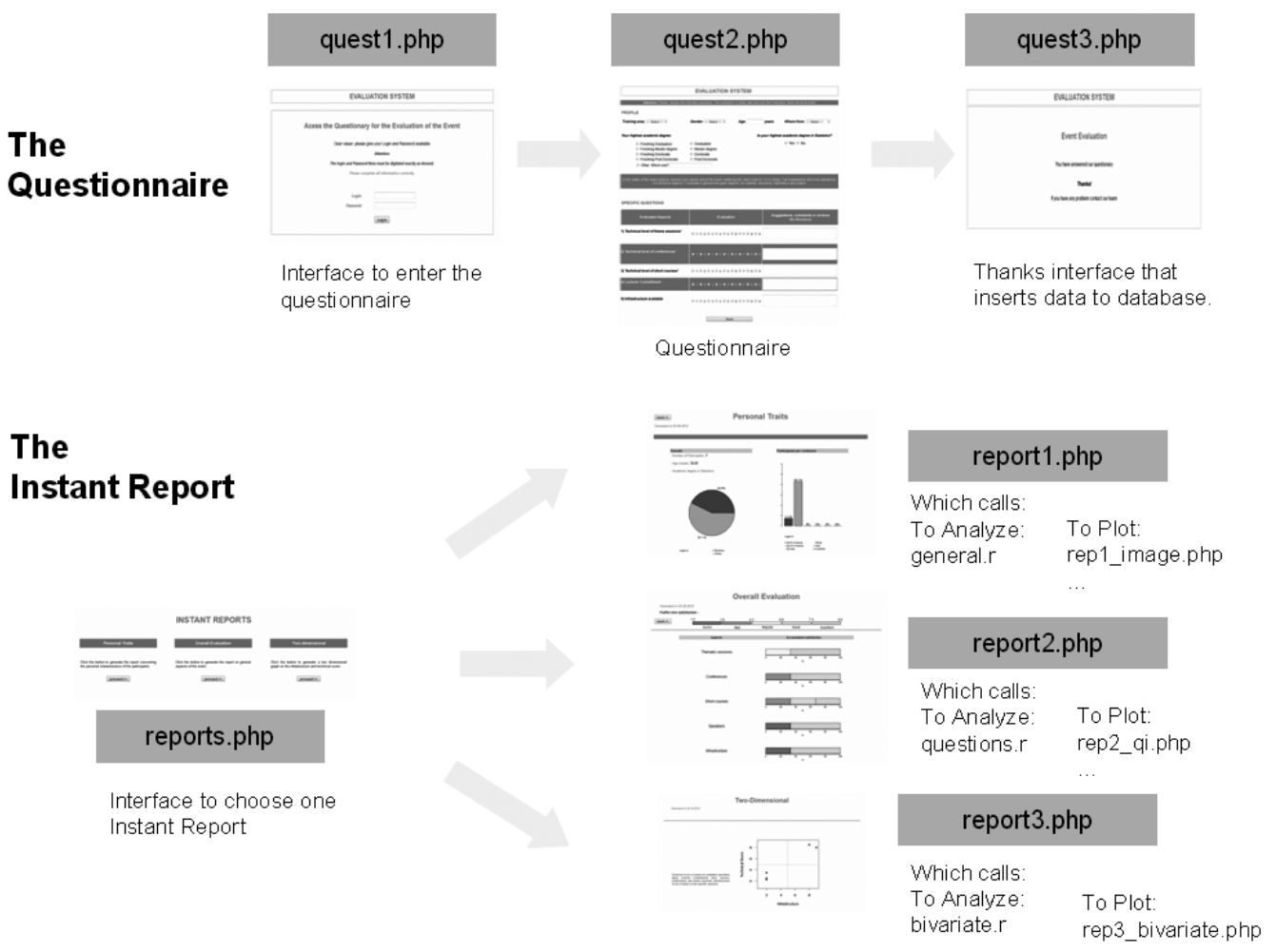

Figure 9 - The files and their relationship in the event evaluation Example.

3. Start the installation, click Install Ubuntu Server.

4. After the detection of the network cards on the computer, we must perform the configuration of the static IP, in other words, the number for the network interface card eth0.

5. Name the server: OnlineSytem.

6. Choose a location: for example, São Paulo, Brazil.

7. After that, we started the disk partitioner through the "assisted partitioning - use entire disk" where in this case, all necessary partitions will be created automatically.

8. Inform the administrator user name and password.

9. Inform the Proxy server address, if any.

10. We must select the packages to be installed along with the environment: the LAMP Server.

11. Once the environment and the chosen services are installed, the server will restart.

After performing the procedures above, the server created can be accessed from any browser (eg. Internet Explorer, Mozilla, Opera etc) through its IP address, showed in Step 4.

We may configure the server directly on the machine installed, by Linux' Shell. Therefore, we are interested in creating a specific place for environment files, making it available through the Internet, installing the $R$ 
software and creating an environment where it can generate graphs and communicate with the MySQL database software.

We start the configuration process by creating a user and their respective folder, where the environment files will be. By taking the user name usertest, use the following command:

sudo useradd usertest

In this case, a folder is created in home / usertest / where the files created by this user will remain, we shall use this folder to save the environment files. Likewise, we can redirect this folder to a fixed address on the Internet. Thus, we edit the alias file with the command:

sudo nano /etc/apache2/conf.d/alias

Notice that in this case we use the nano text editor, which is a traditional editor of the Linux Ubuntu. After opening this file, insert the following lines:

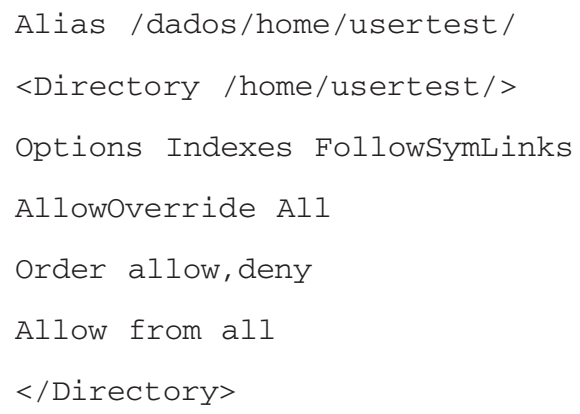

After running the commands above, files that are in the home directory /usertest/ can be accessed directly through a browser using the address IP/data/.

Inside the directory / usertest/ we can create a new directory and save the phpmyadmin files there, where we can manage the tables in MySQL.

In this context, we must install the R software on our server. We must enter the address http://cran. $r$-project.org/bin/linux/ubuntu/ and after that, perform the installation. The entire procedure can be done through the following commands:

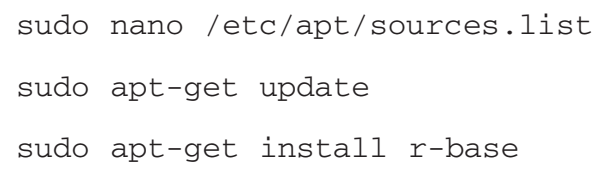

After the construction to this entire structure, we still have to provide the R software with the ability to generate graphics in the Linux environment. We applied ghostscript technology and used the following command line:

sudo apt-get install gs 
We must also provide the R software with the ability to connect with the MySQL database. For such, we used the package RMySQL (James \& DebRoy, 2006), installed with the command:

sudo apt-get install r-cran-rmysql

Directly from the server, we can run the software just by typing $\mathrm{R}$ in any directory. The commands in $\mathrm{R}$ language to access the MySQL database are displayed below (they must necessarily be in .r format and saved on the server):

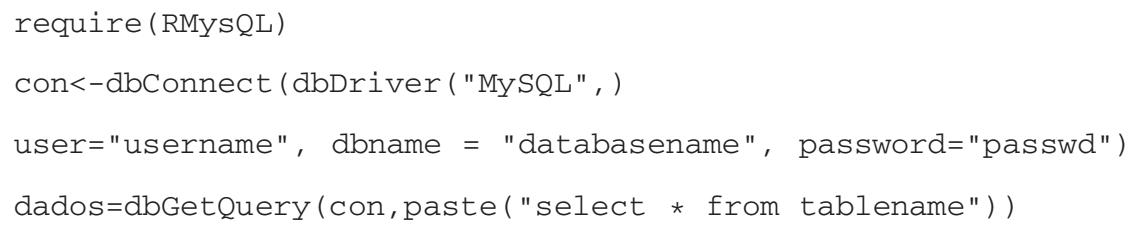

Since, we can edit . php files using the resources installed on the server. By programming in PHP, we can run directly from web pages files written in $\mathrm{R}$ language. The codes below access files . $r$ through the PHP language, statistical.r. This file refers to calculations that have value vectors or images as output. For example, a vector of means, which will be assigned to the variable php \$res or graphs generation codes.

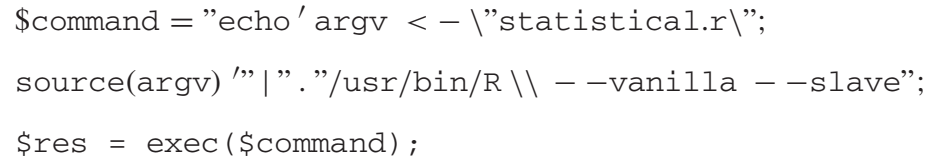

